\title{
LA CLASSE MOYENNE BRÉSILIENNE ET L'ARCHÉTYPE DES PRÉJUGÉS DE CLASSE
}

\section{ARTICLE ORIGINAL}

GIESBRECHT, Daniel Florence ${ }^{1}$

GIESBRECHT, Daniel Florence. La classe moyenne brésilienne et l'archétype des préjugés de classe. Revista Científica Multidisciplinar Núcleo do Conhecimento. An 05, Ed. 02, vol. 04, p. 104-124. février 2020. ISSN: 2448-0959, Lien d'accès: https://www.nucleodoconhecimento.com.br/histoire/archetype-de-prejuge

\section{RÉSUMÉ}

Cet article vise à élaborer une reconstitution historique, de la série à long terme, la formation de la classe moyenne brésilienne et aussi son archétype de préjugés de classe. Nous avons utilisé comme point de départ pour notre réflexion le fait que le Brésil a vécu plus de trois cents ans d'esclavage, qui a légué la profusion d'un imaginaire raciste, résultant en des pratiques préjugées et naturalisé aux populations afrodescendantes, en plus d'avoir étendu au pauvre citoyen, en général. Nous essayons de relier d'une manière historiciste les objets étudiés aux concepts sociologiques de la socialisation, en plus de caractériser les idées bourgeoises de la classe moyenne et les élites brésiliennes de l'histoire des mentalités. Nous avons l'intention de contribuer à une meilleure compréhension des obstacles créés par le manque de pratiques d'altérité dans les relations sociales quotidiennes.

Mots-clés: Classe sociale, préjugés, esclavage, exclusion sociale, altérité.

\footnotetext{
${ }^{1}$ Doctorant en histoire contemporaine; Maîtrise en éducation; Diplôme en sciences sociales, Diplôme en pédagogie, Diplôme d'histoire.
} 


\section{INTRODUCTION}

Lorsque nous avons fait des recherches sur les indicateurs économiques sur le potentiel brésilien, nous avons remarqué des chiffres constants et élevés, très compétitifs par rapport à d'autres pays, à tel point que le Brésil, des années 1960 à aujourd'hui, est toujours parmi les quinze pays ayant le produit intérieur brut le plus élevé au monde.

Les chiffres ne se répètent pas si nous cherchons des données de référence sur les taux de pauvreté et d'inégalité. Les réalités alarmantes de la répartition des revenus, les taux élevés de violence, la mortalité infantile, entre autres, sont facilement perçus aux yeux de ceux qui voyagent à travers le vaste territoire brésilien, donnant l'impression de se promener dans deux mondes complètement différents : celui de l'opulence et celui de la misère, illusion d'optique d'une économie insérée avec succès dans le scénario mondial, mais qui masque une réalité sociale complexe : celle de l'opulence et celle de la misère, illusion d'optique d'une économie insérée avec succès dans le scénario mondial, mais qui masque une réalité sociale complexe , qui présente, dans sa structure, des marques d'inégalités profondes.

Nous utiliserons le mot «pauvreté» pour faire référence à la situation de millions de personnes vivant au Brésil. Mais le concept de pauvreté, selon le contexte utilisé et son affacturage sémantique, devient complexe et polysémique. C'est de cette complexité sémantique que nous proposons d'inférer notre réflexion à travers cet article, qui met en relation la pauvreté avec l'archétype de la formation de la classe moyenne brésilienne et ses interrelations de classe.

Dans l'imaginaire intrinsèque de la classe moyenne brésilienne dans ses cercles de socialisation, l'imagerie de la pauvreté est transfigurée en une perception si simplifiée de la réalité qui les entoure, au point que l'individu de la classe subalterne, souvent dans un état d'insécurité, acquiert inconsciemment le discours de la classe à laquelle elle n'appartient pas et par laquelle elle est exploitée. Dans cette situation, il n'est pas difficile de trouver des phrases quotidiennes du plus pur bon sens qui cherchent, en tout temps, une explication aux maux des inégalités du pays : « II est pauvre parce 
qu'il est vagabond », " Pauvre n'aime pas travailler » ou, pourtant, " Les pauvres ne savent rien faire et ne peuvent rien apprendre ».

Des phrases comme celles-ci finissent par produire une naturalisation du social, c'està-dire qu'elle s'accroche au caractère historique et social d'un fait le réduisant à un événement naturel. Ainsi, quelque chose comme le manque cesse d'être considéré comme le résultat d'une structure assemblée a priori, qui finit par légitimer les inégalités dans sa praxis, d'être considéré comme un attribut personnel d'un individu.

Prenant comme référence l'idée de naturalisation des inégalités et de l'individualisation des attentes et des échecs ponctuels, il est nécessaire de refléter avec précision les facteurs de classe qui contribuent à la naturalisation de l'archétype de l'infériorisation des groupes sociaux désavantagés et qui brise les modèles préconçus depuis l'avènement du positivisme historique du XIXe siècle qui, transformant les sciences sociales en nature régies par des lois immuables qui échappent au contrôle humain , induire le conformisme, l'acceptation passive et résignée de ce qui est produit historiquement et socialement (Sucupira, 1984). L'archétype des pauvres qui échouent au quotidien, si enracinés dans l'imaginaire collectif brésilien de la colonie à la contemporanéité, est en ce sens une opération idéologique, qui sert à maintenir un certain ordre social, c'est-à-dire une configuration de vie économique qui profite aux classes sociales qui produisent cette idéologie.

Nous utiliserons d'abord le cadre théorique de Souza (2019), qui semblait plus pertinent à la critique des paradigmes qui guident la pensée sociale brésilienne, en particulier celle du «culturalisme», dans ses tentatives d'expliquer les origines des inégalités dans le pays. Nous avons également l'intention d'introduire au lecteur les idées que nous défendrons un posteriori, en particulier que la structuration de l'archétype mental de la classe moyenne brésilienne est l'un des principaux obstacles à la construction d'un pays qui consolide les valeurs républicaines, et qui crée des conditions pour l'expansion des droits de citoyenneté pour des millions de ses habitants. 


\section{POLISSÉMIE DE LA PAUVRETÉ}

La pauvreté est un phénomène historique et social. Cela signifie qu'il est étroitement lié à la structure de la société et à la façon dont chaque classe sociale s'approprie les biens produits ou la richesse générée. Ce n'est pas un concept facile, mais nous pouvons utiliser différents critères pour essayer de le définir.

Le critère du revenu personnel ou familial a été le plus utilisé au Brésil, mais nous pensons qu'il est imprécis, en raison de nombreux facteurs qui se sont même aggravés ces dernières années, comme la croissance des activités informelles, qui empêche un contrôle effectif des revenus[2].

En plus du revenu, un autre critère utilisé pour configurer la pauvreté est de vérifier ce qui est considéré comme essentiel pour la reproduction de la vie. Ce critère, donc, à notre avis, est également réductionniste parce que, en raison des conventions internationales, il ne concerne la pauvreté qu'à la consommation alimentaire.

II semble plus pertinent d'étendre le concept aux «besoins fondamentaux», qui ne se limiteraient pas uniquement à la nourriture, mais inclurait d'autres aspects jugés indispensables à la reproduction matérielle de l'existence, élargissant l'idée de pauvreté pour l'incapacité de quelqu'un ou d'une famille à avoir des moyens qui permettent l'accès à un ensemble d'articles considérés comme essentiels à la survie. Cela serait également configuré si la personne ou la famille est exclue du système éducatif, puisque la scolarisation d'aujourd'hui est essentielle pour entrer sur le marché du travail. Selon des études de Rocha (2006), ce n'est qu'avec l'expansion de ces concepts que l'on a pu comprendre un pays de dimensions continentales comme le Brésil, plongé dans d'énormes disparités régionales.

Notre idée argumentative est de surmonter exactement la vision matérielle de la constitution du concept de pauvreté pour une valorisation psychologique et structurelle abstraite et inconsciente qui, dans la construction historique brésilienne, légitime ce concept ancré dans un cadre méthodologique soutenu par l'archétype des préjugés, qui infériorise les groupes sociaux constitués dans un pays qui avait, depuis plus de 
trois cents ans, les marques quotidiennes d'une économie et d'une société orientée au détriment du travail d'esclave.

Les préjugés peuvent servir différents intérêts matériels, qui peuvent être : la classe, le sexe, la politique, la race ou l'origine ethnique et religieuse. Mais pour démontrer la constitution des préjugés naturalisés par la classe moyenne brésilienne, nous avons sans aucun doute besoin de connaître les structures historiques qui ont synthétisé, à travers le passé précapitaliste, les distances entre les groupes sociaux d'une société qui, de la bipolarité entre vous et l'esclave, s'est déplacée vers une organisation plus complexe avec l'avènement du capitalisme commercial et industriel, mais qui a toujours maintenu l'archétype du passé d'esclave.

La discrimination et les préjugés peuvent aussi produire des sentiments de résignation et de conformité chez les victimes, qui acceptent d'être opprimées et exploitées, comme Sartre (1905-1980) l'a averti dans une pièce intitulée The Respectful Prostitut[3]e (1946). II nous semble que le cas brésilien, avec des attitudes quotidiennes de sa classe moyenne d'infériorisation des moins favorisés, les amenant à introjeter leurs propres préjugés et l'idée de l'échec, croyant qu'ils méritent de telles discriminations.

\section{ORIGINES HISTORIQUES DES PRÉJUGÉS DE LA CLASSE MOYENNE BRÉSILIENNE}

Entre le XVle et le XIXe siècle, la traite négrière africaine avec l'Amérique reliait les deux continents à ce qui allait devenir le commerce le plus lucratif de l'histoire brésilienne. Les esclaves africains étaient présents dans les activités quotidiennes les plus diverses. La naturalisation de l'esclavage est devenue quelque chose de si enraciné dans les structures de la société qu'il est très peu probable qu'il soit imaginé sans les divisions traditionnelles de ceux qui commandaient et obéissaient. Ce modèle autoritaire de relations sociales permet de comprendre la permanence symbolique pratiquée contre les populations les plus pauvres, même après l'abolition de l'esclavage à la fin du XIXe siècle. 
Avec le développement de la pensée sociale brésilienne, en particulier depuis le début du XXe siècle, nous avons essayé d'expliquer l'image dichotomère que connaît la société autochtone tout au long de son histoire. Nous croyons que, pour cette tâche, la théorie la plus appropriée, la promotion d'une meilleure compréhension de la construction de cette structure sociale, est celle de l'histoire sérielle, présente dans les méthodologies de Fernand Braude[4]l. II n'est possible d'envisager une analyse sémiotique de la formation des classes sociales brésiliennes que si l'on perçoit la série de la longue durée de l'esclavage, qui a validé dans la mentalité quotidienne du pays l'archétype des préjugés, de la distanciation et de la ségrégation, de la casa-grande à la senzala, qui, avec la transition vers la modernité de la phase mercantile et industrielle du capitalisme, a corroboré une nouvelle forme d'exploitation, mais avec les vêtements structurels de l'ancien, ségréguant riches et pauvres dans les grandes villes, ainsi que dans les milieux sociaux.

Il convient également de rappeler que le développement des classes au Brésil, selon Fausto (1997), est lié à la consolidation d'une classe propriétaire, qui possède la propriété foncière et d'autres moyens de production. Dans un pays autrefois fondamentalement agricole, cette classe s'appropriait facilement la plupart des richesses produites, puisqu'elle exploitait le travail réduit en esclavage.

Sous les auspices de la société coloniale, il s'est développé d'une manière très particulière dans les régions où le système de plantation a été consolidé, les relations sociales en dehors de l'esclavage entre les affranchis et les propriétaires. Avec l'absence d'un système colonial direct, en plus d'une immensité territoriale, qui a entravé la mise en œuvre de formes optimisées de contrôle sous la tutelle de la couronne portugaise, et la création du système de distribution des capitaineries héréditaires - une tentative de conciliation public-privé dans le processus de colonisation - des formes ambigues, contradictoires et déréglementées de relations entre les individus de différentes classes ont été développées. C'est dans ce scénario que nous trouvons le chiffre de l'agrégat.

Cette strate intermédiaire de personnes officiellement libres, entre maîtres et esclaves, est formée d'hommes et de femmes dépendants, à 
la fois matériellement et symboliquement des propriétaires fonciers et des personnes. Au sens le plus économique et matériel, la dépendance de cet extrait social est inexorable. (...) Cela constitue parmi nous comme une forme économique, politique et sociale - la figure de I'"agrégat". Figure fondamentale de la littérature brésilienne et de la sociologie historique, l'agrégat formera la première classe intermédiaire entre propriétaires et dépossédés. (Sousa, 2018: 65-66).

Bien qu'encore éloignés de l'émergence de la classe moyenne brésilienne moderne, le groupe de ménages, même dépendants et épaississant souvent les statistiques de la population dépossédée - la perte de la protection de leur "parrain" était suffisant - il est d'une importance primordiale de comprendre l'introjection mentale des préjugés entre les futures classes sociales du Brésil moderne, étant donné que ceux-ci, dans un système de domination autoritaire du pouvoir privé sur le public , c'est exactement la caste qui a naturalisé les principes de contrôle et de violence pratiqués d'une manière, dans une certaine mesure, sadique par les élites rurales qui travaillent aux esclaves, qui ont également délégué aux agrégats les droits d'opprimer ceux considérés comme des « instruments qui parlaient », bestialisés et déshumanisés.

Le fait que nous intéressions d'ici est de se rendre compte que c'est par cette relation d'interdépendance entre les seigneurs et les agrégats, en plus du monopole de la force établi sous l'État en tant qu'organisateur de la société, que le sentiment de mépris pour l'autre était de conduire à la constitution du comportement sadique avec les esclaves et les plus humbles. Le plaisir de la violence est couramment vérifié dans l'histoire de ces classes intermédiaires dans la société brésilienne, au point d'être plus intense - là nous percevons le sadisme com[5]me une forme de reconnaissance de groupe, ou de supériorité sur l'autre - comme une pratique quotidienne de ceux que nous appelons les hommes libres. Des scènes d'humiliation, de blagues, de satires et de comportements obtus feront partie de l'ampleur du traitement perpétré sur les marginalisés, que nous appellerons la « racaille » sociale.

Depuis le début de l'activité minière dans le crépuscule du XVIle siècle et tout au long du XVIIle siècle, le Brésil a connu le phénomène de l'urbanisation, concentré 
principalement dans la capitainerie du Minas Gerais. La société stratifiée du sucre du Nord-Est était celle d'un personnage plus dynamique et aux multiples facettes à Vila Rica. L'esclavage et la liberté allaient de pair dans une économie interdépendante, mais il a fourni de nouvelles formes d'ascension sociale, y compris les paquebots noir ${ }^{[6]}$ s. L'expérience minière était particulière, dans laquelle le métissage, les Noirs et les Indiens a eu un moment unique dans lequel ils pouvaient mettre en pratique pour la première fois leurs talents, d'où le grand nombre d'artistes, d'artisans, de comptables et l'apparition de plusieurs autres professions dans la région. Pour la première fois dans l'histoire du Brésil, une connaissance technique plus expressive semble se différencier d'une société construite par un travail "brut » et manuel, ouvrant la voie à l'émergence de la classe moyenne moderne.

Le début du 19ème siècle a été le signe avant-coureur de nouveaux airs qui secoueraient le continent européen - et sa plus grande tempête a été Napoléon Bonaparte. Les guerres initiées par l'empereur Français ont été associées, en plus de ses ambitions mégalomaniaques, avec la nouvelle forme assumée par le système capitaliste dans son stade industriel. La France, ennemi historique de la GrandeBretagne, commençait un conflit de proportions continentales pour s'emparer de sa juste part, en présage de ce qui serait le phénomène impérialiste qui serait encore déclenché au milieu du siècle. Ce fait aurait un impact direct et indirect sur l'avenir du Portugal et, par conséquent, du Brésil, parce que la structure administrative métropolitaine traverserait les mers pour s'établir dans la colonie.

Ce processus, que la famille royale portugaise est venu consolider, était déjà prévu dans la découverte des mines, en présence de certaines villes coloniales d'expression, dans la nécessité d'une plus grande vigilance sur la richesse nouvellement découverte et un plus grand contrôle, à partir de là, sur le familismo et le mandonisme privé. Un exemple symptomatique du passage du pouvoir de la campagne aux villes est le cas des dettes des patriarches ruraux jusque-là incollectables, à partir de ce moment-là payés par la police. Aussi important que le passage du centre économiquement dynamique du Nord-Est au Sud-Est a été la 
transformation sociale de grandes proportions, impliquant de nouvelles habitudes, de nouveaux rôles sociaux, de nouvelles professions et, en fin de compte, la construction d'une nouvelle hiérarchie sociale. (Souza, 2019: 61).

L'urbanisation a finalement représenté une resizing des structures de pouvoir, la faisant passer du patriarcat individuel aux mains de l'État qui a été organisé. Du point de vue weberien, le monopole légitime de la violence a commencé à se concentrer sur les mains de l'appareil d'État - comme la police, par exemple - fusionnant avec les prérogatives des anciennes formes d'autoritarisme de classe, mais en maintenant la pratique du sadisme, auparavant privé, maintenant perpétrée par les forces publiques.

Avec l'ouverture des ports aux nations amies en 1808, décrétée par le prince régent de l'époque, $\mathrm{D}$. Joo $\mathrm{VI}$, et avec la signature des traités de commerce et de navigation de 1810, le Brésil connaîtrait des transformations qui auraient immédiatement un impact sur sa structure coloniale. L'environnement des villes portuaires, principalement Rio de Janeiro, Salvador et Recife est devenu, du jour au lendemain, des centres de commerce occupés, avec l'entrée de produits inconnus jusque-là, ce qui a encouragé la formation d'une structure monétaire. Bureaux d'échange et de commerce, agences d'importation et d'exportation, banques, en bref, une multitude d'activités jusque-là inutiles dans le monde du patriarcat rural, seulement connu dans leurs proportions appropriées dans les régions minières[7].

Mais la grande transformation sociale provoquée par ce nouveau scénario européanisé des relations économiques serait l'entrée de milliers d'immigrants. En plus des quelque 15000 Portugais qui sont arrivés ensemble, la famille royale, l'anglais, l'allemand et l'Français sont également venus au Brésil, pour des raisons allant de l'économie à l'art. Le futur pays qui naîtrait en 1822 a déjà marqué les caractéristiques d'un État indépendant.

Les immigrants étaient du travail libre qui, ajouté aux travailleurs libres nés au Brésil, constituerait la classe moyenne moderne dont nous avons parlé plus tôt. Une classe différente de l'ancien ménage rural, occupant la partie intermédiaire de la hiérarchie 
sociale, mais sans avoir besoin de la protection de son compadre. Les principes libéraux et l'individualisme, qui soufflaient depuis le XVIIle siècle avec la philosophie des Lumières, sont entrés dans l'inconscient de l'citadin. Une sorte d'homme auto fait des tropiques peuplerait l'imaginaire de la ville.

Avec le capitalisme mercantile ouvre un vide fondamental pour son maintien et sa reproduction : la nécessité d'un capital culturel pour l'exploiter. Les activités commerciales, manufacturières, bureaucratiques et pédagogiques, qui en sont quelques exemples, exigent une forme de connaissances techniques et utilitaires pour leur fonctionnement, un fait qui contribuerait à l'opposition du travail manuel, jusqu'ici couramment pratiqué par l'esclave ou les plus pauvres. La classe moyenne sera le groupe qui s'appropriera cette connaissance et, à travers elle, se distanciera des plus humbles, ceux du travail lourd, du niveau des bêtes.

Pour les abandonnés et les oubliés, les tâches disqualifiées, typiques de la racaille, restent. Ceux-ci, dans une large mesure, comme dans le cas du travail domestique, seront une continuation de l'esclavage, maintenant sous de nouveaux masques. L'exploitation directe de l'énergie musculaire - qui nous distingue à peine d'un cheval ou d'une mule - avec peu de connaissances incorporées, est la caractéristique distinctive, sinon la seule, par rapport aux travailleurs ayant un degré plus élevé de qualification technique. (Souza, 2018: 74).

Tant cette nouvelle classe moyenne, que la future classe ouvrière mini-spécialisée, que l'on appellera "la classe moyenne basse", ou "prolétarienne", tenteront à tout prix de se différencier de la population considérée comme "racaille", formée principalement par les Noirs et les métis. Comme ils n'ont pas les moyens de production, ils tenteront au moins d'imiter les valeurs symboliques des élites, en réfléchissant à l'obsession de l'européanisation des coutumes et, par l'éducation formelle et technique, à la distanciation du travail manuel, si dépréciée par les valeurs occidentales. Ici, nous avons notre première manifestation concrète de préjugés intégré par la classe moyenne moderne du 19 ème siècle. 
Les questions endogènes et exogènes par rapport à la question des esclaves brésiliens ont été déterminantes pour la maturation initiale du processus de modernisation des relations de travail et de la maturation du capitalisme industriel au XIXe siècle. Depuis l'indépendance du Brésil, l'Angleterre fait pression sur l'État national nouveau-né pour qu'il démontre ses engagements en faveur de l'extinction progressive de la traite négrière pratiquée depuis le milieu du XVle siècle. Les mouvements internes dirigés par des intellectuels libéraux, comme l'élite du café ellemême, débattaient ouvertement de l'abolition.

De tels faits, dans un premier temps, pourraient indiquer d'une manière sui generis que le pays, à son rythme, pourrait consolider sa transition du capitalisme commercial au capitalisme industriel, parce que le scénario qui s'est ouvert avec l'abolition de l'esclavage déplacerait non seulement une énormité du capital vers les secteurs productifs, mais aussi la modernisation des relations de classe, surmontant les amalgames du système esclavagiste obsolète. Mais ce n'est pas ce qui s'est passé. L'industrialisation ne se consolidera qu'au siècle suivant, sous la direction de l'État, dans les gouvernements Getlio Vargas et Juscelino Kubitschek, ainsi qu'à la période dictatoriale. Surmonter l'esclavage, dans son domaine symbolique, ne serait jamais surmonté.

Même avec l'abolition consommée en 1888, un fait qui a fait du Brésil le dernier pays au monde à mettre fin à l'esclavage formel, le scénario pervers de la violence physique et symbolique s'est poursuivi. En outre, les Noirs libérés ont rencontré d'énormes difficultés dans leur insertion sur le marché du travail, car ils devaient encore rivaliser avec la main-d'œuvre immigrée, maintenant délibérément stimulée comme pratique de l'État. C'est dans cette relation perverse de permanence que, avec le temps, le Brésil a été formé, en même temps, avec le processus d'urbanisation, une classe de la population qui a été complètement exclue des principes fondamentaux de la citoyenneté, et ce[8]la affecterait également ses générations futures, un effet que nous appellerons la « boule de neige trouble[9]». 


\section{À L'EXCLUSION DE L'INDUSTRIALISATION ET DE L'IMAGINAIRE BOURGEOIS DE LA CLASSE MOYENNE}

L'ancien ordre racial n'a pas disparu avec la fin du travail des esclaves. Au lieu de cela, il a été assimilé et intégré dans les nouvelles structures sociales découlant du développement du travail libre. Selon Fernandes (1974), la révolution bourgeoise déclenchée avec la fin de l'esclavage ne profitait que de quelques segments de la société brésilienne.

Parmi les plus favorisés se trouvait la partie " blanche » de la population d'origine européenne, corroborant les théories du blanchiment en vogue dans le pays. Ce groupe a bénéficié de toutes les transformations résultant de la croissance économique, de la poussée d'industrialisation, du processus d'urbanisation et du développement d'une culture urbaine. La classe marginalisée, composée d'Afrodescendants, est restée en marge de la société nationale, exclue en grande partie de toutes les améliorations produites par ces transformations. Ces faits souliquaient que la désarticulation du travail des esclaves dans les dernières décennies du XIXe siècle ne s'accompagnait pas de changements substantiels dans l'ancien ordre racial, caractérisé par des préjugés et de la discrimination. La marginalisation sociale d'environ $50 \%$ des Brésiliens a clairement mis en évidence les limites de la modernisation économique du pays.

Avec le maintien des moyens de production entre les mains de l'agro-exportelite et de la classe industrielle naissante, c'est dans la ville et dans son environnement cosmopolite que le mode de vie bourgeois s'est consolidé et, avec lui, tout son symbolisme. C'est avec l'émergence de la classe moyenne urbaine, en particulier celle axée sur les activités intellectuelles et financières, que le sentiment d'européanisation et de coutumes s'est répandu. L'incorporation des valeurs aristocratiques européennes peut être perçue dans l'adoption d'un modèle de goût et de luxe qui a cherché à correspondre à celui des couches aristocratiques. Dans une sorte de « cannibalisme symbolique ", la classe moyenne la plus riche introjectait les préjugés et le patriarcat, mais avec un vêtement que l'on disait moderne. 
Dans une dialectique symbolique, la classe moyenne est l'un des meilleurs éléments pour nous de percevoir comment la sphère publique et privée étaient fondamentales à leur affirmation de soi, leur reconnaissance et leur distinction. Même loin du concept moderne de " méritocratie ", on pe[10]rçoit comment l'archétype des plus " bien adaptés » résonne dans l'imaginaire du bourgeois moyen. Le mérite a remplacé le droit d'aînesse comme une nouvelle forme de différenciation sociale, ainsi que la sophistication intellectuelle, un autre élément qui a commencé à distinguer la classe moyenne des classes populaires, toujours sans accès à une éducation publique gratuite et de qualité.

La généralisation de la famille de la classe moyenne, dans laquelle la femme s'occupait de ses enfants et des tâches ménagères - contrôlant le travail de nombreux employés - et l'homme a participé aux activités du monde des affaires, est l'un des aspects frappants en ce sens, car elle donne à l'individu le sentiment de continuité, voyant chez ses enfants la possibilité de continuer avec l'entreprise familiale. Par conséquent, l'incorporation de valeurs aristocratiques, plus qu'une simple mode, faisait partie du processus de consolidation de l'hégémonie politique et sociale et de la vision du monde de la classe moyenne sur la sociét[11]é.

Une fois que l'univers symbolique bourgeois de la classe moyenne brésilienne est établi, il est clair la perception antagoniste de celui-ci envers les autres groupes moins aisés et leur tentative de s'équiper symboliquement avec les élites, ces plutôt véritables détenteurs des moyens de production et le contrôle réel du pays. Cette obsession de ressembler aux élites ne sera pas, sauf dans certains cas, reconnue dans la sphère monétaire, donc, son équivalence symbolique par le statut socia[12]I ou le prestige reste, et cela peut être obtenu ou reconnu par trois éléments: l'occupation productive (emploi), la consommation et le mode de vie, tous entièrement adaptés à la société capitaliste moderne, ou, plus que cela , l'un complétant l'autre.

Avec la conquête du statut dans la société méritocratique brésilienne moderne, de nouvelles formes de prestige font partie de la nouvelle hiérarchie sociale. La chose la plus importante pour la classe moyenne, en plus de la «vertu» de l'imitation, est de se distancier des pratiques quotidiennes de la «racaille» sociale. Boire du vin au détriment 
de la cachaça, écouter de la musique classique au lieu de la musique populaire, assister à des théâtres, des académies littéraires et des restaurants coûteux pour l'exhibitionnisme de l'étiquette, en plus des voyages internationaux - avec le droit de conseiller de pseudointellectuals, se nourrissant du sentiment ambigu de révulsion à ce qui est national - sont de petits exemples de cette vision du monde réductionniste.

\section{CAPITAL CULTUREL, INSTITUTIONS SOCIALES ET PRÉJUGÉS}

Contrairement au point de vue culturaliste identifié au début de notre texte comme un moyen d'expliquer les inégalités sociales et les relations de classe, les concepts de subjectivation et de justifier les différences uniquement du point de vue matériel individuel, nous devons partir de la compréhension sociologique des institutions primaires et secondaires de socialisation pour comprendre le phénomène de la haine de classe si présent dans la vie quotidienne.

En général, la socialisation commence dans la famille, une institution dans laquelle l'individu apprend la langue et, à travers elle, de communiquer, d'exprimer des besoins, ainsi que des sentiments d'inconfort ou de bien-être. La recherche psychologique comme le piaget (2005) confirme l'importance des parents et des autres membres de la famille dans la formation de la personnalité des enfants. Ces études indiquent qu'au début du processus de socialisation, les membres de la famille ont tendance à être plus décisifs que les personnes avec lesquelles l'enfant n'entre en contact qu'occasionnellement. Nous trouvons ici un problème: dans l'organisation de la société esclavagiste brésilienne tout a été fait pour éviter la construction de liens de solidarité entre les populations asservies, et la première institution combattue a été la famille, compromettant cette étape de la socialisation. Il est à noter qu'il n'y avait guère de place pour la constitution des liens sociaux primaires en prison, sans tenir compte des besoins psychiques et émotionnels des captifs.

La difficulté de l'organisation familiale n'est pas un simple phénomène du passé des esclaves, mais demeure, en proportion, parmi les populations les plus humbles, dans les enfants, petits-enfants et arrière-petits-enfants des anciennes générations de dégradants. Tout cela aura des conséquences profondes dans les futures relations 
secondaires, en particulier dans l'apprentissage scolaire, ce qui aura moins de chances de prestige sur le marché du travail. Les familles les plus faibles sont généralement taxées comme « inadaptées » par les classes supérieures, en particulier par le discours généraliste de la classe moyenne, qui, dès le berceau, a su construire des liens de solidarité qui seront déterminants pour le succès futur.

Le cas actuel de l'exploitation de la racaille brésilienne pour gagner du temps des tâches ménagères sales et lourdes - qui vous permet d'utiliser le temps "volé" à un prix ignoble dans des activités plus productives et bien payées - montre une fonctionnalité de misère claire comme la lumière du soleil. Cette lutte de classe silencieuse exempte toute une classe de garde d'enfants et de vie à domicile, transformant l'argent de gain de temps et l'apprentissage admissible. La classe volée, dans ce cas, est condamnée éternellement à jouer les mêmes rôles laïques serviles. (Souza, 2019: 85).

Gagnez du temps sur les tâches manuelles et investissez du temps dans des activités intellectuelles. Par conséquent, une plus grande formation professionnelle et, par conséquent, un plus grand prestige social; un cercle est établi qui n'a rien à voir avec le mérite, ou la capacité personnelle, mais plutôt avec l'appropriation d'un plus grand capital culturel.

Selon Bourdieu (2004), les enfants issus de familles aisées ont tendance à avoir des résultats scolaires plus élevés que les jeunes issus de familles appartenant aux classes populaires. Dans la plupart des cas, c'est parce que les élèves des classes dominantes ont un capital culturel que d'autres n'ont pas pu accumuler, non seulement à l'école, mais aussi dans la formation culturelle des parents, qui enseigneraient à leurs enfants les exigences de maintenir et d'élargir leur situation sociale privilégiée. Les enfants en situation de fragilité sociale n'ont pas ce réseau de protection, qui ont tendance à faire face à beaucoup plus de difficultés parce qu'ils n'ont pas les connaissances et les pratiques valorisées par la culture dominante. Ils ne participeraient pas aux valeurs cultivées par ces élites et, pour cette raison, auraient tendance à considérer l'apprentissage scolaire comme une forme de violence contre 
leurs valeurs et pratiques sociales, expliquant les taux élevés de décrochage de l'école brésilienn[13]e.

À l'école, nous avons peut-être la meilleure perception pratique de la violence symbolique. Par exemple, il est courant pour les élèves des classes populaires de rencontrer une langue, une pratique culturelle et des valeurs qu'ils ne connaissent pas parce qu'ils n'ont pas un certain patrimoine culturel, ou, comme le souligne Bourdieu, un capital culturel qui leur permet de les comprendre ou de les assimiler, de compromettre leur performance scolaire, de devenir un obstacle imposé par l'institution elle-même et de délégitimer la performance de cet étudiant. Malgré cela, l'élève ne se rend pas compte que son faible rendement scolaire est déterminé par des facteurs sociaux et finit donc souvent par inintroduire l'échec, compromettant son estime de soi à un moment crucial dans sa formation non seulement académique mais humaine.

Ayant comme point de référence l'accès et la continuité dans le système éducatif formel comme l'une des principales chances proclamées par une société qui se considère comme «méritocratique», nous trouvons un exemple subtil de la façon dont les limitations imposées par le passé d'esclave sont perpétuées, mais presque invisibles. Si dans le passé nous pouvions percevoir tout explicitement inégal, en raison du phénotype et de l'exclusion explicite des esclaves vers les institutions, aujourd'hui tout semble corrigé en raison de la création d'un système d'éducation publique, en transférant toutes les responsabilités de réussite ou d'échec à la portée individuelle. II couvre le soleil avec le tami[14]s, parce que la plupart des populations dans le besoin sont exclues du capital culturel, délibérément approprié par la classe moyenne et les élites, en plus d'avoir accès à un système de service public inefficace.

Dans le monde capitaliste, le manque de succès matériel le condamne au statut d'échec. Imaginez-vous alors dépourvu de capital matériel et aussi de capital culturel ? Parce que les formes d'ascension de classe dans le système prédateur du capital ne sont pas faciles, le cycle reproducteur de la pauvreté et de l'exclusion tend à se perpétuer de la naissance à ceux qui viennent des classes les plus pauvres. Cela se traduira par le sujet, en plus de moins de chances de constituer une formation qui lui 
permettra de rivaliser avec les enfants des classes riches, beaucoup plus susceptibles de subir toutes sortes de violence physique et symbolique.

Comme la reproduction de l'inégalité des classes du berceau est réprimée consciemment et inconsciemment, c'est le stéréotype du noir, facilement reconnaissable, qui identifie facilement l'ennemi à massacrer et à exploiter. Le "danger noir» utilisé comme mot de passe pour massacrer sans défense et quilombola pendant des siècles se poursuit par d'autres moyens dans le massacre ouvert, et aujourd'hui applaudi sans pejo, de pauvres et de noirs dans les bidonvilles et les prisons. Et pas seulement ça. Comme il n'y avait pas de continuité temporelle entre l'esclavage - qui détruit l'âme de l'intérieur, humilie et rabaisse le sujet, faisant de lui un complice de sa propre domination - et la production d'une racaille d'inadaptés au monde moderne, nos exclus hérités, sans solution de continuité, toute haine et mépris lâche pour les plus faibles et avec moins de capacité à se défendre. (Souza, 2019: 88).

Mais pourquoi une telle inégalité ne semble-t-elle pas perçue par la classe moyenne brésilienne ? Comme nous l'avons dit au début de cet article, la théorie des classes sociales prises par des critères de revenu est insuffisante pour comprendre une composition sociale complexe comme celle du Brésil, affaiblie par les conséquences de son passé d'esclave. À notre avis, il est nécessaire de surmonter le simple biais économique pour entrer dans l'histoire des mentalités[15]. Un citoyen de la classe moyenne a tendance à le considérer comme sa classe, de sorte qu'il n'aurait aucune base instrumentale pour la perception de l'autre, ce qui rend l'exercice de l'altérité misérable ou souvent inaccessible. Cette absence de dialectique produit des personnes préjugées, dépourvues de perception de l'autre, dans un pays inséré au XXle siècle dans un ordre de marché concurrentiel, qui va au-delà de la sphère économique, atteignant le social, la pensée et la perception du monde. Le colonialisme mental finit par dominer les esprits tout en maintenant le statu quo. 


\section{CONSIDÉRATIONS FINALES}

Retracer une reconstitution historique de la formation de la classe moyenne brésilienne et son archétype des préjugés n'est pas une tâche facile en raison de l'ambiguïté du concept de classe sociale elle-même. Le facteur que nous croyons essentiel pour cette compréhension est l'esclavage passé, qui interfère considérablement dans la compréhension du présent et du tissu social brésilien. $\mathrm{Ce}$ n'est que dans la série à long terme que nous pouvons construire le récit présenté ici et notre argumentation pour la compréhension des classes du contexte socioculturel, rompant avec l'économie traditionnelle des théories de classe, parfois interprétées par des penseurs libéraux, parfois par des penseurs marxistes.

Identifiant l'importance du passé d'esclave pour la configuration des mentalités de classe perpétuées par les institutions sociales, nous pouvons percevoir les inégalités et les stéréotypes socialement constitués qui semblent reproduire ad infinitum.

La famille, l'école, les organisations, tout cela promeut tout au long de notre histoire la reproduction des privilèges des groupes élitistes et de la classe moyenne, qui est camouflée avec l'encre d'analyses pseudoscientifiques de la méritocratie, dans l'un des pays les plus inégalitaires de la planète. L'erreur qu'il est possible que l'élévation presque innée pour certains au détriment des autres a été incorporée dans l'imaginaire de la classe moyenne si régulièrement qu'elle a réussi à pénétrer même les couches populaires, dans le phénomène que nous traitons dans le texte sur l'introjection des préjugés.

Le fait d'être né dans la classe moyenne et la classe moyenne supérieure est presque synonyme de reproduction du succès des générations passées et de l'incorporation de privilèges qui feront une différence totale à l'avenir. Ils semblent être des faits insignifiants, mais l'acquisition dès le plus jeune âge de la discipline de l'étude, la fréquentation de certains environnements, la vie avec les personnes avec le capital culturel nécessaire pour exceller dans les relations sociales, l'étude d'une langue étrangère dans le quart d'école et d'être bien nourri sont de petits exemples de 
privilèges qui, ajouté à des centaines d'autres, façonnera le futur citoyen réussi, comme si son mérite personnel avait été le grand différentiel de cette réalisation.

La naturalisation de la violence quotidienne contre les pauvres, les Noirs, les femmes, entre autres minorités, est symptomatique de la violence qui a façonné l'histoire brésilienne. Les échos de l'esclavage sont entendus dans chaque mort dans la favela, dans toutes les agressions commises par la police, dans chaque blague au déjeuner du dimanche racontée aux tables des familles qui ont déjà naturalisé le racisme comme quelque chose moralement acceptable, comme s'il n'était pas humiliant la dignité humaine, transformant ainsi la réalité de la classe moyenne en une cage de peurs, d'angoisse et de haine systémique.

\section{RÉFÉRENCES}

BONFIM, Paulo Ricardo. Educar, higienizar e regenerar: uma história da eugenia no Brasil. São Paulo: Paco, 2019.

BOURDIEU, Pierre. A economia das trocas simbólicas. São Paulo: Perspectiva, 2004.

BRAUDEL, Fernand. Escritos sobre a história. São Paulo: Perspectiva, 1978.

CARVALHO, José Murilo de. Cidadania no Brasil: o longo caminho. São Paulo: Civilização Brasileira, 2003.

DOWBOR, Ladislau. A era do capital improdutivo. São Paulo: Editora Novas Palavras, 2017.

FAUSTO, Bóris. História do Brasil. 6. ed. São Paulo: Edusp/FDE, 1997. . História concisa do Brasil. São Paulo :Edusp, 2002.

FERNANDES, Florestan. Revolução burguesa no Brasil: ensaio de interpretação sociológica. Rio de Janeiro: Zahar, 1974.

FROMM, Erich. Anatomia da destrutividade humana. Rio de Janeiro: Zahar, 1975. 
KENNETH, Maxwell. A devassa da devassa. São Paulo: Paz e Terra, 1995.

KLEIN, Naomi. Sem logo: a tirania das marcas em um planeta vendido. Rio de Janeiro: Record, 2002.

PARSONS, Talcott. Social structure \& person. USA: Free Press, 2007.

PIAGET, Jean. A representação do mundo na criança. Curitiba: Ideias e Letras, 2005.

ROCHA, Sônia. Pobreza no Brasil. Rio de Janeiro: FGV, 2006.

SARTRE, Jean Paul. A prostituta respeitosa. Campinas: Papirus, 1992.

SOUZA, Jessé. A classe média no espelho. Rio de Janeiro: Estação Brasil, 2018. A elite do atraso. Rio de Janeiro: Estação Brasil, 2019.

SUCUPIRA, Eduardo. Introdução ao pensamento dialético. São Paulo: Alfa-Omega, 1984.

VOVELLE, Michel. Ideologias e mentalidades. São Paulo: Brasiliense, 1987.

WEBER, Max. A ética protestante e o espírito do capitalismo. São Paulo: Companhia das Letras, 2007.

YOUNG, Michael. The rise of meritocracy. United Kingdom: Transaction Pub, 1994 


\section{ANNEXE A}

\begin{tabular}{|c|c|c|c|c|c|c|c|c|c|c|}
\hline \multirow{3}{*}{ Grandes Regiôes e car acterísticas selecionadas } & \multicolumn{10}{|c|}{$\begin{array}{l}\text { Taxa de frequência liquida a estabelecimento de ensino da populacão residente de } 6 \text { a } 24 \text { anos de idade, por grupos de idade e nivel de } \\
\text { ensino (\%) }\end{array}$} \\
\hline & \multicolumn{2}{|c|}{$\begin{array}{l}6 \text { a } 14 \text { anos, no ensino } \\
\text { fundamental }\end{array}$} & \multicolumn{2}{|c|}{$\begin{array}{l}6 \text { a } 10 \text { anos, nos anos } \\
\text { iniciais do ensino } \\
\text { fundamental }\end{array}$} & \multicolumn{2}{|c|}{$\begin{array}{l}11 \text { a } 14 \text { anos, nos anos } \\
\text { finais do ensino } \\
\text { fundamental }\end{array}$} & \multicolumn{2}{|c|}{$\begin{array}{l}15 \text { a } 17 \text { anos, no ensino } \\
\text { médio }\end{array}$} & \multicolumn{2}{|c|}{$\begin{array}{l}18 \text { a } 24 \text { anos, no ensino } \\
\text { superior }\end{array}$} \\
\hline & Taxa & CV $(\%)$ & 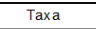 & $\mathrm{CV}(\%)$ & 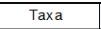 & $\mathrm{CV}(\%)$ & 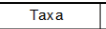 & $\mathrm{CV}(\%)$ & 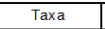 & $\mathrm{CV}(\%)$ \\
\hline Brasil & 97,0 & 0,1 & 95,5 & 0,2 & 85,9 & 0,3 & 68,5 & 0,6 & 23,2 & 1,3 \\
\hline Norte & 96,6 & 0,2 & 95,0 & 0,4 & 79,4 & 1,0 & 59,7 & 1,8 & 17,7 & 3,3 \\
\hline Nordeste & 96,8 & 0,1 & 95,3 & 0,2 & 82,0 & 0,6 & 60,7 & 1,1 & 16,8 & 2,9 \\
\hline Sudeste & 97,3 & 0,2 & 95,7 & 0,3 & 89,2 & 0,5 & 76,5 & 1,0 & 25,8 & 2,4 \\
\hline Sul & 97,4 & 0,2 & 96,1 & 0,3 & 89,6 & 0,6 & 69,6 & 1,4 & 29,1 & 2,4 \\
\hline Centro-Oeste & 96,8 & 0,3 & 95,0 & 0,4 & 88,4 & 0,9 & 70,4 & 1,7 & 30,1 & 2,9 \\
\hline Situaçäo do dom icilio & & & & & & & & & & \\
\hline Urbana & 97,1 & 0,1 & 95,5 & 0,2 & 87,2 & 0,3 & 70,6 & 0,7 & 25,6 & 1,4 \\
\hline Rural & 96,9 & 0,2 & 95,6 & 0,2 & 79,6 & 0,7 & 57,4 & 1,3 & 8,3 & 3,5 \\
\hline Sexo & & & & & & & & & & \\
\hline Homem & 96,9 & 0,1 & 95,3 & 0,2 & 83,5 & 0,5 & 63,6 & 0,9 & 19,7 & 1,8 \\
\hline Mulher & 97,2 & 0,1 & 95,7 & 0,2 & 88,3 & 0,4 & 73,7 & 0,7 & 26,8 & 1,5 \\
\hline Cor ou raça (1) & & & & & & & & & & \\
\hline Branca & 97,3 & 0,1 & 95,8 & 0,2 & 89,5 & 0,4 & 76,6 & 0,8 & 32,9 & 1,6 \\
\hline Preta ou parda & 96,9 & 0,1 & 95,3 & 0,2 & 83,7 & 0,4 & 63,5 & 0,9 & 16,7 & 1,8 \\
\hline $\begin{array}{l}\text { Classes de percentual de pessoas em ordem } \\
\text { crescente de rendimento domiciliar per capita }\end{array}$ & & & & & & & & & & \\
\hline Até 20\% & 96,6 & 0,2 & 94,9 & 0,2 & 78,9 & 0,6 & 54,7 & 1,3 & 6,6 & 4,5 \\
\hline Mais de $20 \%$ até $40 \%$ & 96,9 & 0,2 & 95,4 & 0,3 & 85,3 & 0,6 & 65,3 & 1,2 & 12,0 & 3,3 \\
\hline Mais de $40 \%$ até $60 \%$ & 97,5 & 0,2 & 96,1 & 0,3 & 90,1 & 0,6 & 73,9 & 1,0 & 20,1 & 2,6 \\
\hline Mais de $60 \%$ até $80 \%$ & 97,5 & 0,3 & 95,9 & 0,5 & 93,0 & 0,6 & 81,1 & 1,2 & 32,3 & 2,1 \\
\hline Mais de $80 \%$ & 97,8 & 0,3 & 96,1 & 0,5 & 95,0 & 0,5 & 90,7 & 0,8 & 58,3 & 1,5 \\
\hline
\end{tabular}

\section{ANNEXE B}

Distribuição percentual da população, por classes de percentual de pessoas em ordem crescente de rendimento real efetivo domiciliar per capita e cor ou raça, com indicaçáo do coeficiente de variaçao, se gundo as Grandes Regibes e as Unid

\begin{tabular}{|c|c|c|c|c|c|c|c|c|c|c|c|c|}
\hline \multirow{3}{*}{$\begin{array}{l}\text { Grandes Regiōes e } \\
\text { Unidades da Fede raçäo }\end{array}$} & \multicolumn{12}{|c|}{ Distribuição percentual da população, por classes de percentual de pessoas em ordem crescente de rendimento real efetivo domiciliar per capita e cor ou raça (\%) } \\
\hline & \multicolumn{4}{|c|}{ Total } & \multicolumn{4}{|c|}{ Entre os $10 \%$ com menores rendimentos } & \multicolumn{4}{|c|}{ Entre os $10 \%$ com maiores rendimentos } \\
\hline & \multicolumn{2}{|c|}{ Branca } & \multicolumn{2}{|c|}{ Preta ou parda } & \multicolumn{2}{|c|}{ Branca } & \multicolumn{2}{|c|}{ Preta ou parda } & \multicolumn{2}{|c|}{ Branca } & \multicolumn{2}{|c|}{ Preta ou parda } \\
\hline Brasil & 43,6 & 0,5 & 55,4 & 0,4 & 23,9 & 1,8 & 75,2 & 0,6 & 71,7 & 0,8 & 26,3 & 2,3 \\
\hline Norte & 20,1 & 1,9 & 78,4 & 0,5 & 12,5 & 6.8 & 84,8 & 1,3 & 34.2 & 3,8 & 64,4 & 1,9 \\
\hline Acre & 19,1 & 4,3 & 79,9 & 1,1 & 14,1 & 12,5 & 85,3 & 2,1 & 31,8 & 8,2 & 67,7 & 3,8 \\
\hline Amazonas & 18,1 & 4,7 & 79,5 & 1,2 & 11,0 & 13,1 & 86,2 & 2,0 & 38,2 & 9,8 & 60,5 & 5,8 \\
\hline Roraima & 21,5 & 5,6 & 72,4 & 2,0 & 14,6 & 17,7 & 71,3 & 7,3 & 40,9 & 8,4 & 55,5 & 6,5 \\
\hline Pará & 18,2 & 3,3 & 80,6 & 0,8 & 11,8 & 12,6 & 85,8 & 2,2 & 30,0 & 5,5 & 68,9 & 2,4 \\
\hline Nordeste & 24,8 & 1,2 & 74,6 & 0,4 & 18,7 & 3,3 & 80,8 & 0,8 & 42,8 & 2,7 & 56,4 & 2,0 \\
\hline Maranhão & 18,5 & 2,6 & 80,8 & 0,6 & 15,7 & 6,9 & 83,9 & 1,3 & 31,5 & 5,5 & 67,4 & 2,6 \\
\hline Pauí & 20,5 & 4,8 & 79,4 & 1,2 & 18,6 & 11,0 & 81,2 & 2,5 & 40,9 & 7,6 & 58,6 & 5,5 \\
\hline Ceará & 27,5 & 2,2 & 71,9 & 0,8 & 20,4 & 6,2 & 79,1 & 1,6 & 46,9 & 4,3 & 52,6 & 3,8 \\
\hline Rio Grande do Norte & 36,7 & 2,6 & 63,1 & 1,5 & 30,6 & 7,1 & 69,1 & 3,1 & 50,9 & 5,5 & 48,8 & 5,8 \\
\hline Paraba & 33,8 & 3,0 & 66,1 & 1,5 & 30,1 & 7,2 & 69,8 & 3,1 & 54,1 & 5,4 & 45,7 & 6,3 \\
\hline Pernambuco & 30,7 & 3,0 & 68,5 & 1,4 & 23,2 & 8,3 & 76,5 & 2,5 & 51,5 & 5,8 & 47,4 & 6,2 \\
\hline Alagoas & 23,4 & 2,8 & 76,1 & 0,9 & 21,8 & 6,3 & 77,9 & 1,8 & 36,1 & 6,6 & 63,5 & 3,8 \\
\hline Rio de Janeiro & 43,8 & 1,7 & 55,7 & 1,3 & 28,4 & 5,2 & 71,2 & 2,1 & 74,5 & 1,9 & 24,8 & 5,8 \\
\hline São Paulo & 60,1 & 1,2 & 38,1 & 2,0 & 46,8 & 3,7 & 52,4 & 3,3 & 83,1 & 1,4 & 12,3 & 9,0 \\
\hline Sul & 75,6 & 0,5 & 23,7 & 1,7 & 59,3 & 2,1 & 39,9 & 3,2 & 91,3 & 0,6 & 7,9 & 6,9 \\
\hline Paraná & 67,2 & 1,1 & 31,7 & 2,3 & 52,2 & 3,8 & 46,7 & 4,3 & 86,7 & 1,3 & 11,4 & 9,4 \\
\hline Santa Catarina & 82,8 & 0,7 & 16,9 & 3,4 & 68,5 & 2,9 & 31,2 & 6,4 & 92,8 & 0,9 & 6,8 & 11,7 \\
\hline Rio Grande do Sul & 79,6 & 0,9 & 20,0 & 3,5 & 64,2 & 3,3 & 35,2 & 6,1 & 94,4 & 0,9 & 5,5 & 15,4 \\
\hline Centro-Oeste & 36,3 & 1,4 & 62,6 & 0,8 & 25,0 & 4,5 & 74,0 & 1,5 & 56,2 & 3,4 & 41,0 & 4,0 \\
\hline Mato Grosso do Sul & 43,2 & 2,6 & 55,5 & 2,1 & 28,9 & 8,7 & 69,1 & 3,7 & 65,0 & 3,6 & 34,1 & 6,8 \\
\hline Mato Grosso & 31,8 & 3,2 & 67,0 & 1,5 & 23,7 & 12,3 & 74,9 & 3,9 & 54,4 & 4,8 & 43,9 & 6,0 \\
\hline Goiás & 35,7 & 2,2 & 63,6 & 1,2 & 23,9 & 7,4 & 75,8 & 2,3 & 51,6 & 4,2 & 47,7 & 4,6 \\
\hline Distrito Federal & 36,7 & 4,1 & 61,6 & 2,4 & 22,8 & 11,0 & 76,5 & 3,3 & 59,3 & 10,5 & 29,1 & 10,0 \\
\hline
\end{tabular}

Fonte: IBGE Pesquisa Nacional por Amostra de Domichilos Contínua, 2017, consolidado de primeiras entrevistas .

Notas: Exclusive as pessoas cuja condição no arranjo domiciliar era pensionista, empregado doméstico ou parente do empregado doméstico.

Rendimentos deflacionados para reais médios do próprio ano.

Não são apresentados resultados para amarelos, indigenas e pessoas sem declaração de cor ou raça.

Disponível em: https://www.nucleodoconhecimento.com.br/histoire/archetype-de-prejuge 


\section{ANNEXE C - RÉFÉRENCES NOTE DE BAS DE PAGE}

2. Para o entendimento da financeirização do capitalismo e suas repercussões no mundo do trabalho, vide DOWBOR, Ladislau. A era do capital improdutivo. São Paulo: Editora Novas Palavras, 2017.

3. SARTRE, Jean Paul. A prostituta respeitosa. Campinas: Papirus, 1992. Dans cette pièce, l'auteur décrit comment l'introjection des préjugés, incorporée par la victime, est naturalisée au sein de la société du sud des États-Unis.

4. BRAUDEL, Fernand. Escritos sobre a história. São Paulo: Perspectiva, 1978.

5. FROMM, Erich. Anatomia da destrutividade humana. Rio de Janeiro: Zahar, 1975.

6. Uma das obras mais consistentes e rica em fontes primárias sobre a organização das regiões mineradoras é de KENNETH, Maxwell. La débauche de la débauche. São Paulo: Paz e Terra, 1995.

7. Sobre o período em questão, vide FAUSTO, Bóris. História concisa do Brasil. São Paulo: Edusp, 2002.

8. Para um melhor entendimento sobre a construção do processo da cidadania brasileira, vide CARVALHO, José Murilo de. Cidadania no Brasil: o longo caminho. São Paulo: Civilização Brasileira, 2003.

9. Nous avons métaphoriquement utilisé l'expression « boule de neige trouble », parce que les Noirs, le métis, les indigènes, les bruns, en plus bien sûr de la population blanche marginalisée, épaissit le contingent de «scories » séparées de la société hiérarchique brésilienne. Au XIXe siècle, le racisme scientifique et les principes de l'eugénisme que l'on retrouve en Amérique, en particulier chez les eugénistes américains, ses principaux porte-parole. Bien que les préjugés au Brésil soient limités à tort aux questions phénotypiques, le rôle joué par les eugénistes dans la stimulation des politiques de blanchiment, délibérément assumées par le gouvernement brésilien afin de purifier la génétique de la population, est considérable. Cela a contribué 
directement à la stimulation du racisme et de l'animalisation des personnes considérées comme «inférieures ». BONFIM, Paulo Ricardo. Educar, higienizar e regenerar: uma história da eugenia no Brasil. São Paulo: Paco, 2019.

10. Au Brésil, le concept de «méritocratie» imprègne le bon sens et la démagogie. À notre avis, il n'est compris que la « méritocratie " si nous entrons dans sa littérature dans laquelle le concept est né d'une manière satirique et ironique. Ver YOUNG, Michael. The rise of meritocracy. United Kingdom: Transaction Pub, 1994.

11. "Avec l'avènement de la culture de masse au XXe siècle, le capitalisme crée un nouveau symbolisme de fausse inclusion, introduisant dans la société contemporaine ce que nous appelons la "suppression de la subjectivité", soulevant le développement du soi-disant "comportement mimétique", c'est-à-dire que l'individu aurait cessé de développer une personnalité qui lui est propre, originale, qui le mettrait en conflit et en tension avec la réalité sociale établie." KLEIN, Naomi (2009). Sem logo: a tirania das marcas em um planeta vendido. Rio de Janeiro: Record, 2002.

12. Tomamos como referência o conceito de status em WEBER, Max. L'éthique protestante et l'esprit du capitalisme. São Paulo: Companhia das Letras, 2007.

13. Selon les annexes $A$ et $B$, nous pouvons clairement noter les maux historiques du processus d'exclusion des populations noires et brunes dans leur parcours éducatif, distançant ces groupes des mécanismes d'accès requis par la société contemporaine pour une vie digne, qui exige un meilleur placement professionnel, compromettant leurs revenus et leur capacité à entrer dans le monde capitaliste.

14. Expression populaire brésilienne utilisée pour décrire des mesures inefficaces pour certains problèmes.

15. VOVELLE, Michel. Ideologias e mentalidades. São Paulo: Brasiliense, 1987.

Envoyé: Décembre, 2019.

Approuvé : février 2020. 